\title{
Management of Nasopharyngeal Carcinoma in Elderly Patients
}

\author{
Wing Lok Chan ${ }^{1}$, James Chung Hang Chow ${ }^{2}$, Zhi-yuan $X u^{3}$, Jishi $L i^{3}$, \\ Wing Tung Gobby Kwong ${ }^{1}$, Wai Tong $\mathrm{Ng}^{1}$ and Anne W. M. Lee ${ }^{1 *}$ \\ ${ }^{1}$ Department of Clinical Oncology, The University of Hong Kong, Pokfulam, Hong Kong SAR, China, ${ }^{2}$ Department of Clinical \\ Oncology, Queen Elizabeth Hospital (QEH), Hong Kong SAR, China, ${ }^{3}$ Department of Clinical Oncology, Shenzhen Hospital, \\ University of Hong Kong, Shenzhen, China
}

\section{OPEN ACCESS}

Edited by:

Jan Baptist Vermorken,

University of Antwerp, Belgium

Reviewed by:

Tianzhu Lu,

Jiangxi Cancer Hospital, China

Naomi Kiyota,

Kobe University Hospital, Japan

Yungan Tao,

Gustave Roussy Cancer Campus,

France

${ }^{*}$ Correspondence: Anne W. M. Lee awmlee@hku.hk

Specialty section: This article was submitted to Head and Neck Cancer, a section of the journal

Frontiers in Oncology

Received: 07 November 2021 Accepted: 03 January 2022 Published: 01 February 2022

Citation:

Chan WL, Chow JCH,

Xu Z-y, Li J, Kwong WTG,

Ng WT and Lee AWM (2022)

Management of Nasopharyngeal

Carcinoma in Elderly Patients.

Front. Oncol. 12:810690.

doi: 10.3389/fonc.2022.810690
Nasopharyngeal cancer (NPC) is one of the most difficult cancers in the head and neck region due to the complex geometry of the tumour and the surrounding critical organs. High-dose radical radiotherapy with or without concurrent platinum-based chemotherapy is the primary treatment modality. Around $10 \%-15 \%$ of NPC patients have their diagnosis at age after 70 . The management of NPC in elderly patients is particularly challenging as they encompass a broad range of patient phenotypes and are often prone to treatmentrelated toxicities. Chronologic age alone is insufficient to decide on the management plan. Comprehensive geriatric assessment with evaluation on patients' functional status, mental condition, estimated life expectancy, comorbidities, risks and benefits of the treatment, patients' preference, and family support is essential. In addition, little data from randomized controlled trials are available to guide treatment decisions in elderly patients with NPC. In deciding which treatment strategy would be suitable for an individual elderly patient, we reviewed the literature and reviewed the analysis of primary studies, reviews, and guidelines on management of NPC. This review also summarises the current evidence for NPC management in elderly adults from early to late stage of disease.

Keywords: nasopharyngeal carcinoma, elderly, geriatric assessment, frailty, chemotherapy, radiotherapy

\section{BACKGROUND}

Nasopharyngeal carcinoma (NPC) is characterised by its unique and distinct geographical distribution, with $70 \%$ cases in east and southeast Asia. Because of its challenging location below the skull base limiting surgical accessibility, radiotherapy (RT) is the primary treatment modality. Although the classical non-keratinizing type is radiosensitive, a high-radiation dose is needed for achieving locoregional control. Furthermore, the combination of RT with concurrent plus induction or adjuvant chemotherapy is recommended for patients with advanced disease. The management of NPC in the elderly population is particularly challenging. Elderly patients are at a higher risk of toxicity from anticancer treatment due to comorbidities, as well as suboptimal nutritional status, organ function, and/or social support as compared to younger patients (1-4). There is lack of treatment guidelines for optimal management for the elderly. This review highlights the specific consideration in assessment in elderly patients with NPC and summarises the current evidencebased treatment landscape. The age distribution of NPC is unique: the relative risk increases with 
age and peaks at approximately 55 years of age and begins to decline at ages over 60 years. According to the Hong Kong Cancer Registry, the proportion of new cases of NPC with age $\geq$ 70 ranged from $10.6 \%$ to $14.4 \%$ (5).

Majority of NPC patients as a whole presented with advanced disease. Two studies showed that the stage distribution pattern of elderly NPC is similar to that of the general population $(5,6)$. In Hong Kong, the overall stage distribution at presentation was as follows: stage I $6.5 \%$, stage II $14.1 \%$, stage III $40.7 \%$, and stage IV $29.8 \%$ (7). For patients aged $\geq 70$ years, $50.6 \%$ had stage III disease while $25.2 \%$ had stage IV disease. In China, according to the National Cancer Data Base (NCDB), over $63.1 \%$ of patients aged $>65$ years had stage III and IV disease (8). In Singapore, similarly, $73.4 \%$ of elderly NPC patients had stage III or IV disease (9).

With advanced disease at presentation, together with presence of multiple comorbidities and suboptimal functional status, most clinicians would use less aggressive treatment in elderly patients. Previous studies reported that elderly patients with NPC had a worse treatment outcome compared with the overall population. In a matched cohort study, despite similar rates of complete locoregional response, survival of NPC patients aged $\geq 65$ who underwent chemoradiotherapy was significantly lower than that of demographic-matched young-age control (5year OS, $75.3 \%$ vs. $54.6 \%$; 5 -year CSS, $77.8 \%$ vs. $56.6 \%$ ) (10). In the study by Huang et al. on 1,137 NPC patients aged $\geq 65$, the 5 year OS was only $50.4 \%$, which is lower than that of the general population of over $60 \%$ (8). Age over 75 was one of the independent prognostic factors in OS [hazard ratio (HR) 1.951, 95\% confidence interval (CI) $1.552-2.453$, p-value $<0.001]$. The study by Liang et al. also found that elderly patients had significantly worse 5-year OS compared with younger patients [5-year OS: $82.7 \%$ (age $60-<65$ ) vs. $60.9 \%$ (age $65-<70$ ) vs. $46.2 \%$ (age $\geq 70), \mathrm{p}$-value $=0.002]$ and progression-free survival (PFS) [5-year PFS: $74.3 \%$ (age $60-<65$ ) vs. $60.9 \%$ (age $65-<70$ ) vs. $46.5 \%$ (age $\geq 70$ ), p-value $=0.002$ ] (11). Sommat et al. similarly showed that age $>70$ had significant negative impact on OS (HR 2.40, 95\% CI 1.44-4.00, p-value 0.001) and disease-free survival (DFS) (HR 1.85, 95\% CI 1.02-3.36, p-value 0.043) (9).

\section{CHALLENGES FACED BY ELDERLY PATIENTS WITH NPC}

\section{Limited Data From Prospective Studies}

Despite the increasing number of the elderly population with cancer, elderly individuals were often underrepresented in clinical studies. In the MAC-NPC meta-analysis, those aged $\geq 60$ years attributed only $13 \%$ of the total cohort (12). Patients aged 70 years and above are often excluded from clinical studies which often selected participants with better performance status and general condition. Moreover, the age cut-off of "elderly" is not universally defined. Previous prospective studies on NPC selected 60-65 years as the cut-off point for the elderly NPC population. The general health in 60-65 years is improving globally, making the choice of this age cut-off arguable.
Currently, the cut-off of 70 years is the most commonly adopted cut-off for defining patients as "elderly" in the field of geriatric oncology. In addition, many elderly patients do not meet the eligibility criteria of clinical trials, due to comorbidities, reduced organ functions, and/or worse performance status. The optimal treatment of elderly patients (aged $\geq 70$ ) remains unclear. The existing treatment guidelines for management of NPC are based on studies on non-elderly patients, limiting the applicability in the elderly population.

\section{Comorbidities}

Smoking is one of the risk factors for development of NPC (13). It is also associated with other comorbidities, including chronic obstructive pulmonary disease, cardiovascular disease, renal impairment, gastrointestinal disorders, and metabolic syndrome. Elderly patients with NPC, at the same time, have a higher incidence of these comorbidities. There are different validated assessment tools to evaluate comorbidities in elderly patients, e.g., Charlson Comorbidity Index (CCI) and the Adult Comorbidity Evaluation-27 (ACE-27) instrument (ACE-27). Both tools are shown in Appendix I. The incidence of comorbidity in elderly patients with NPC ranged from $22.4 \%$ to $58 \%$. The study by Huang et al. reported comorbidities present in $22.4 \%$ of patients using the Charlson Comorbidity Index (CCI) (8). Ramakrishnan et al. using the Adult Comorbidity Evaluation-27 (ACE-27) instrument reported a 44\% incidence of comorbidities in 59 patients with NPC, with cardiovascular and pulmonary diseases as most common (14). Guo et al. demonstrated that $44.2 \%$ patients with NPC in southern China had comorbidity using ACE-27, with the most common comorbidity being gastrointestinal disease (15). The wide difference in incidence of comorbidity is partly due to the use of different indices to measure comorbidities. ACE-27 identified more comorbidities than CCI as ACE-27 captures additional pancreatic, neuromuscular, psychiatric, and a wider range of cardiovascular diseases, alcohol and illicit drug use, and obesity information not captured by CCI (16).

Medical comorbidity has been reported to have a strong relationship with survival and outcome. Huang et al. demonstrated that presence of comorbidity was significantly associated with worse OS (8). Patients with a CCI score of 0 had significantly higher 5 -year OS than patients with a CCI score of 1 or $\geq 2(53.1 \%$ vs. $42.2 \%$ vs. $32.9 \%, p<0.001)$. In the multivariate analysis, CCI was a statistically significant independent prognostic factor for the risk of death of all causes for patients with a CCI score of 1 (HR 1.242; 95\% CI $1.002-1.539$ ) or CCI score of $\geq 2$ (HR: 1.625 ; 95\% CI: $1.157-$ 2.283) when compared to patients with a CCI score of 0 . The study by Sze et al., which included 103 patients aged $>70$, revealed that ACE-27 was a significant prognostic factor for OS (5-year OS: $48.5 \%$ for ACE-27 score $0-1$ vs. $20.6 \%$ for ACE score $2-3, \mathrm{p}=0.003$ ) and cancer-specific survival (CSS) (5-year CCS: $71.1 \%$ for ACE-27 score $0-1$ vs. $53.0 \%$ for ACE score $2-3$, $\mathrm{p}=0.02$ ) (6). The study by Jin et al. on 126 NPC patients aged 70 or above treated with intensity-modulated radiotherapy (IMRT) showed that ACE-27 was significantly associated with survival outcomes (5-year OS: $72.9 \%$ in ACE-27 score $0-1$ vs. $39.9 \%$ in 
ACE-27 score 2-3, $\mathrm{p}=0.001)(17)$. Guo et al. also showed that comorbidity was a significant independent prognostic factor for OS and DFS. Patients with ACE-27 score $>1$ had a significantly better OS compared with those with score $\leq 1$ (5-year OS $81.1 \%$ vs. $59.3 \%, \mathrm{p}<0.001$ ) and better DFS (5-year DFS: $74.1 \%$ vs. $43.9 \%, \mathrm{p}<0.001)(15)$. Yang et al. similarly demonstrated that a higher CCI score was associated with worse survival (18).

In addition, elderly patients usually have poor nutritional status, immobility, and cognitive decline, leading to impaired tolerability to treatment and higher risk of adverse events compared with the younger population.

\section{Assessment of Elderly Cancer Patient With NPC}

All these considerations cautioned against using chronological age alone in risk-stratifying patients for management for NPC. Both the International Society of Geriatric Oncology (SIOG) and the American Society of Clinical Oncology (ASCO) recommend comprehensive geriatric assessment (CGA) to develop individually tailored cancer care plans for elderly patients (19, 20). CGA is regarded as the gold standard in the assessment of frailty. It is a multidimensional and multidisciplinary evaluation of elderly patients' physical functioning (multimorbidity, mobility/ falls, nutrition, polypharmacy, and sense [sight and hearing]), psychological health (cognition and mood), functional status (activities of daily living and instrumental activities of daily living), and social well-being (21). It allows the clinical team to identify risk factors such as dementia, malnutrition, and poor social support, which may compromise the tolerability of treatment, and hence individual tailoring of recommendations to optimize cancer treatment and follow-up. Table 1 shows the important domains of assessment in elderly patients with cancer. For example, NPC patients with poor nutritional status have poor prognoses and worse overall survival (22). Early nutritional intervention can improve nutritional status, increase tolerance to cancer treatment, improve QoL, and prolong survival. Depression is another poor prognostic factor for elderly patients with NPC (23). Early management of depression would help patients to better tolerate the side effects of treatment. Patients with multiple comorbidities also have more acute toxicities in chemoradiotherapy; omission of chemotherapy may have to be considered.

Although CGA is recommended in different international oncology guidelines, it is not widely implemented because it is time consuming and there is a shortage of trained staff. Geriatric screening tools have been developed and can be used to identify those who will benefit most from a geriatric assessment. To date, Geriatric-8 (G8) and Vulnerable Elders Survey-13 (VES-13) were the two most commonly used tools for identifying patients who need CGA (24). The details of G8 and VES-13 are shown in Appendix 3 and 4. Studies also showed that G8 could predict survival. The study by Pottel et al. (25) demonstrated that vulnerability as classified by G8 was an independent predictor for survival after curative chemoradiotherapy for elderly patients with head and neck cancer (25). Ishii et al. also showed that an abnormal G8 score in elderly patients with head and neck cancer was significantly associated with a shorter survival, higher 30-day mortality, and all-complication rates (26). These studies showed that the geriatric screening tool not only can help identify those patients who need comprehensive assessment but also can predict complication and treatment outcome.

TABLE 1 | Important domains in geriatric assessment.

\begin{tabular}{|c|c|c|}
\hline $\begin{array}{l}\text { Geriatric assessment } \\
\text { domain }\end{array}$ & Examples of evaluation tools & Suggested interventions \\
\hline Functional status & $\begin{array}{l}\text { Instrumental activities of daily living (The Katz Index of Independence in Activities of } \\
\text { Daily Living, Lawton Instrumental Activites of Daily Living Scale), } \\
\text { Physical performance (ECOG performance status, KPS) }\end{array}$ & Prehabilitation \\
\hline Nutrition & $\begin{array}{l}\text { Body mass index } \\
\text { Weight loss (kilogram loss in } 2 \text { months) } \\
\text { Swallowing problem }\end{array}$ & $\begin{array}{l}\text { Dietary counselling } \\
\text { Nutritional supplement }\end{array}$ \\
\hline Cognition & Cognitive screening (Mini-cog), decision making capacity & Complete neuropsychological evaluation \\
\hline Comorbidities & $\begin{array}{l}\text { Number of comorbidities } \\
\text { (e.g., Charles Comorbidity Index, ACE-27) }\end{array}$ & Referral to relevant specialties \\
\hline Polypharmacy & $\begin{array}{l}\text { Number of medications used } \\
\text { Any inappropriate use of medications } \\
\text { Compliance of medications } \\
\text { Any drug interactions with anti-cancer treatment }\end{array}$ & Refer pharmacy to review the medications used \\
\hline Falls & History of falls (number of falls in the past 6 months) & $\begin{array}{l}\text { Physiotherapy, fall risk assessment, home } \\
\text { environment modification, use of walking aids }\end{array}$ \\
\hline $\begin{array}{l}\text { Life expectancy } \\
\text { calculation }\end{array}$ & Actuarial tables, personalized calculators & $\begin{array}{l}\text { Better communication platform with patients and } \\
\text { carers }\end{array}$ \\
\hline $\begin{array}{l}\text { Treatment toxicity } \\
\text { calculation }\end{array}$ & Geriatric assessmet-bsed calculators, e.g., CARG toxicity tool, CARSH score & $\begin{array}{l}\text { Treatment dose adjustments, need of any } \\
\text { prophylactic treatment }\end{array}$ \\
\hline Social support & $\begin{array}{l}\text { Number of carers at home in daytime and night time } \\
\text { Capacity of carers to take care of the patient }\end{array}$ & Referral to social worker if needed \\
\hline $\begin{array}{l}\text { Psychological } \\
\text { assessment }\end{array}$ & Depression/anxiety (geriatric depression scale, HADS) & $\begin{array}{l}\text { Referral to clinical psychologist } \\
\text { Use of medications }\end{array}$ \\
\hline
\end{tabular}

ECOG, Eastern Cooperative Oncology Group; KPS, Karnofsky Performance Status; HADS, Hospital Anxiety and Depression Scale; ACE-27, Adult Comorbidity Evaluation-27; CARG, The Cancer and Aging Research Group; CRASH, Chemotherapy Risk Assessment Scale for High-Age Patients. 


\section{TREATMENT OF NPC IN ELDERLY PATIENTS}

\section{Definitive Treatment in Non-Metastatic Disease}

The current standard of care for non-metastatic NPC is highdose radiotherapy using the intensity-modulated technique (IMRT) and incorporation of systemic chemotherapy in patients with locoregionally advanced tumours. Despite the improvement of radiotherapy techniques, treatment of NPC remains a harsh journey even for young and fit individuals.

We have performed a literature review on the management plan of elderly patients with NPC. A suggested management flow is also shown in Figure 1.

\section{Radiotherapy}

NPC is highly sensitive to ionising radiation; treatment outcomes have been excellent in contemporary series in the era of IMRT (27). Past studies on RT with the 3D conformal technique showed that elderly NPC patients had poor tolerance to highdose radiotherapy. Sze et al. showed that patients aged $\geq 70$ had significantly higher rates of grade 3-4 acute radiation mucositis (68\% vs. $57.6 \%)$ and dermatitis $(22.3 \%$ vs. $12.7 \%)$ than the younger counterpart (6). Furthermore, elderly patients had a significantly higher 90-day mortality rate compared with younger patients $(7.8 \%$ vs. $1.2 \%, \mathrm{p}<0.001)$. This early mortality was associated with poor comorbidities: patients with a pretreatment Adult Comorbidity Evaluation-27 (ACE-27) score of 2-3 have a 15 -fold higher risk of early deaths than those scored $<2$.
With advances in the radiotherapy technique, intensitymodulated radiotherapy (IMRT) or tomotherapy has become the standard RT technique for NPC. IMRT provides excellent tumour target coverage and allows the delivery of a high dose to the target with significant sparing of the organs at risk, e.g., salivary glands, brainstem, and cranial nerves $(28,29)$. Studies demonstrated that IMRT achieved better local control with fewer late toxicities compared with the $3 \mathrm{D}$ conformal technique. Recent studies demonstrated that elderly NPC patients treated with IMRT had similar toxicity profiles compared with others. Only few patients could not complete radiotherapy, with incidence ranging from $0 \%$ to $2.7 \%(9,30)$. The most common severe toxicities (grades 3-4) were dermatitis and mucositis $(9,11)$.

With improving survival following contemporary definitive treatments, late complications have become an important and relevant domain in survivorship care. A retrospective matched cohort study did not identify a significant difference in the incidences of late radiotherapy complications between elderly and young NPC patients. Specifically, advanced age did not increase the risk of radiation-associated sensorineural hearing loss (31, 32), neurocognitive decline (33), cranial neuropathy (34, $35)$, and impairment in overall quality of life $(32,36,37)$. Interestingly, elderly NPC patients even seemed to be less susceptible to develop post-radiation hypothyroidism $(38,39)$. On the contrary, elderly NPC patients are more prone to develop post-radiation xerostomia and second malignancy $(40,41)$. In a recent territory-wide study in Hong Kong, the risk of second cancer in post-IMRT NPC patients aged $>60$ was $40 \%$ higher compared with the demographic-matched general population. The absolute excess cancer risk in this elderly age group was up

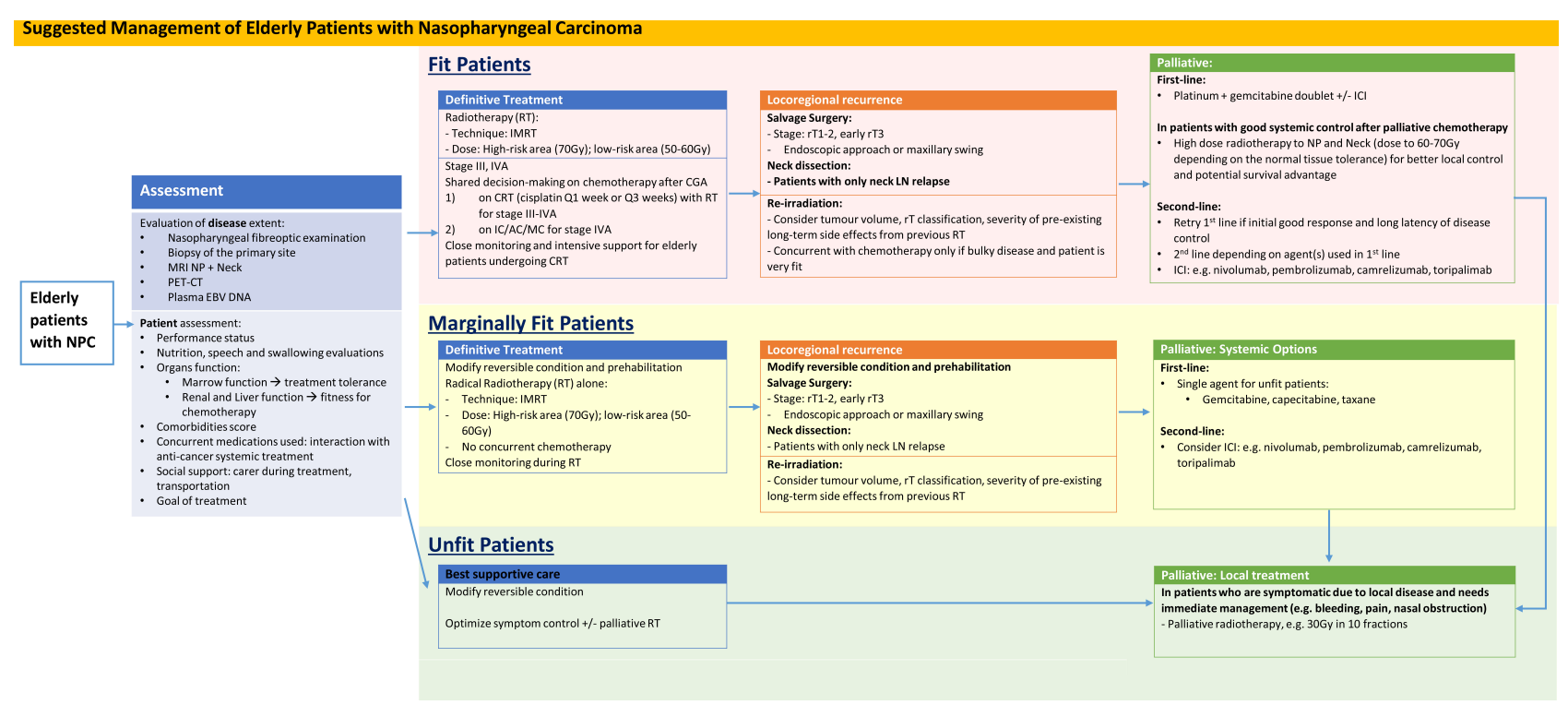

FIGURE 1 | Suggested Management of Elderly Patients with Nasopharyngeal Carcinoma. AC, adjuvant chemotherapy; CRT, concurrent chemoradiation; IC, induction chemotherapy; ICl, immune checkpoint inhibitors; IMRT, intensity modulated radiotherapy; LN, lymph node; MC, metronomic chemotherapy; NP, Nasopharynx; RT, Radiotherapy. 
to 60 per 100,000 person-years at risk. As such, continual surveillance for radiation-associated second cancer is warranted in elderly NPC survivors (34).

Despite serious concern about the higher rates of early mortality and acute radiation toxicities, currently there is a lack of clinical evidence to support de-escalation of radiation dose or volume for elderly NPC patients. Radiotherapy planning for elderly NPC should follow international guidelines on target delineation and dose prioritization, maintaining prescribed radiation doses to high-risk and low-risk at 70 and 50-60 Gy, respectively $(42,43)$. A retrospective study using simultaneous integrated boost to gross nasopharyngeal tumour to $68 \mathrm{~Gy}$ in 30 fractions showed a satisfactory long-term outcome in elderly NPC patients, but $20 \%$ of patients had unplanned radiotherapy interruptions due to high-grade mucositis and hematologic toxicities (44). Compared with IMRT, intensity-modulated proton has demonstrated dosimetric advantages and a decreased rate of gastrostomy tube insertion with therapy in localised NPC $(45,46)$. However, its long-term therapeutic value and cost effectiveness in elderly patients remain to be elucidated by prospective comparative research.

\section{Chemotherapy}

Since the first report of the milestone Intergroup 0099 showing survival benefit by concurrent-adjuvant chemotherapy in the late 1990s, incorporating systemic chemotherapy has become the standard recommendation for locoregionally advanced NPC. The MAC-NPC meta-analysis which combined results of 19 trials has concluded a $6 \%$ absolute improvement in 5-year OS with the addition of chemotherapy to definitive radiotherapy (12). Both the latest ESMO-EURACAN and CSCO-ASCO guidelines recommend concurrent plus induction or adjuvant chemotherapy for advanced-stage diseases $(47,48)$.

When applying clinical evidence to elderly NPC patients, it must be emphasized that this population is highly underrepresented in most clinical trials. According to population incidence data from the Hong Kong Cancer Registry, $44.7 \%$ of NPC patients diagnosed between 2009 and 2018 were aged $\geq 60$ years (7), whereas the same age group only constituted $13.0 \%$ of the 4,806 patients included in MAC-NPC (12).

The therapeutic benefit of concomitant chemotherapy in elderly NPC patients has remained uncertain. Retrospective data on elderly patients (age $\geq 60$ or $\geq 65$ ) with advanced NPC suggested improvements in survival outcomes with the addition of chemotherapy to 2-dimensional radiotherapy, particularly in patients with low pretreatment comorbidities $(10,49)$. Nevertheless, in the MAC-NPC meta-analysis, a clear decrement in the hazard ratios for chemotherapy on OS was observable as age increases: 0.72 for age $<50,0.79$ for age $50-59$, and 0.89 for age $\geq 60$, suggesting a diminished therapeutic advantage for chemotherapy in elderly NPC patients (12). This observation is in line with that of the MACH-HC meta-analysis in head and neck squamous cell carcinoma, in which no clear benefit of chemoradiotherapy was found in patients aged $\geq 70$, and the authors have recommended concomitant chemotherapy to be weighed carefully in the elderly age group (50). In the contemporary era of IMRT, multiple retrospective studies which focused in elderly NPC patients reported higher toxicities with concomitant chemotherapy, e.g., myelosuppression, nausea, vomiting, and mucositis $(5,9,17,44,51)$. Hence, consideration of adding chemotherapy for elderly patients should be individualized.

Cisplatin has remained the core cytotoxic agent for treating NPC. Despite its well-proven efficacy, cisplatin is notorious for causing ototoxicity, nephrotoxicity, and peripheral neuropathy, all of which are especially worrisome for elderly patients with limited organ reserve. Expert consensus on head and neck cancer patients aged $>70$ at high risk of cisplatin toxicities raised the suggestion that extra care should be taken before administration (52). Understanding that a dichotomized cut-off by chronological age is oversimplified, CGA should be considered before using cisplatin. In selected elderly NPC patients with good organ functions, concomitant chemotherapy may still be a reasonable option after careful comorbidity assessment. In one study, the benefit of concomitant chemotherapy was confined to patients with ACE-27 scores of $<2$, whereas all patients with ACE-27 score $\geq 2$ who underwent chemoradiotherapy experienced grade 3-4 acute toxicities (10).

Concurrent cisplatin is most commonly administered in a schedule of $100 \mathrm{mg} / \mathrm{m}^{2}$ every 3 weeks. Randomized studies have demonstrated similar efficacy of the weekly $30-40-\mathrm{mg} / \mathrm{m}^{2}$ regimens, which require less fluid pre-hydration and potentially less renal and otologic toxicities $(53,54)$. However, preliminary data from a phase III non-inferiority trial in NPC demonstrated significantly higher rates of grade 3-4 thrombocytopenia and leukopenia with the weekly regimen than the tri-weekly regimen (55). Although elderly patients could attain a higher cumulative cisplatin dose with the weekly regimen than the tri-weekly regimen (median, 240 vs. $160 \mathrm{mg} /$ $\mathrm{m}^{2}$ ) (10), prospective evidence on the safety profile of the weekly cisplatin regimen in the elderly population is lacking.

Several studies have compared alternative platinum derivatives with conventional cisplatin in advanced NPC. Carboplatin, nedaplatin, and lobaplatin have been independently compared with cisplatin in phase III clinical trials, in which all have demonstrated non-inferior progression-free survival, with improved quality of life and less ototoxicity, nephrotoxicity, and emetogenicity (56-58). However, the upper-bound age limits for patients in these trials were set at 70,65, and 60 years, respectively. It has therefore remained uncertain whether the efficacy and favourable toxicity profiles of these agents would be maintained in the elderly NPC population. Nimotuzumab, an anti-epidermal growth factor antibody, has also been studied in elderly NPC in one retrospective study (59). Nimotuzumab demonstrated encouraging efficacies and tolerability but incurred relatively high rates of grade 3-4 leukopenia and mucositis, when used with or without concomitant cisplatin in advanced elderly NPC patients ( $\geq 60$ years old) undergoing definitive radiotherapy. Prospective phase II trials are ongoing to investigate this approach, with special focus in the elderly NPC population (ClinicalTrials.gov Identifier: NCT03025958, NCT03915132).

Based on the landmark Intergroup 0099 study and three subsequent confirmatory trials, definitive chemoradiotherapy 
followed by 3 cycles of 5 -fluorouracil and cisplatin in the adjuvant phase is one of the standard recommendations for advanced NPC (60-63). However, as patients are still experiencing significant acute toxicities after chemoradiotherapy, the adjuvant component of this regimen is often poorly tolerated, with only approximately $60 \%$ of patients being able to complete all three cycles. Administration of adjuvant chemotherapy is particularly challenging in elderly patients $(9,17,51)$. A recent study showed that metronomic capecitabine $\left(650 \mathrm{mg} / \mathrm{m}^{2}\right.$ body surface area twice a day) for 1 year, compared with observation, significantly improved failure-free survival in patients with highrisk locoregionally advanced NPC (3-year failure-free survival: $85.3 \%$ vs. $75.7 \%$, HR $0.50,95 \%$ CI $0.32-0.79, \mathrm{p}=0.0023)$ with a manageable safety profile (64). However, the study did not include patients aged $>65$ years.

In recent years, several phase III clinical trials have demonstrated survival advantages of induction chemotherapy before definitive chemoradiotherapy in NPC (65-67). However, elderly patients were excluded in these trials due to anticipated concern about tolerance. In a propensity score-matched analysis focused on NPC patients aged $\geq 60$, the addition of induction chemotherapy has no significant impact on survival compared to chemoradiotherapy alone but led to worse hematologic toxicities and stomatitis (68). With the current lack of evidence, the therapeutic benefit of concurrent with/without adjuvant or induction chemotherapy in elderly NPC patients remains uncertain, the risks should be duly discussed, and close monitoring and intensive support should be provided for elderly patients treated with additional chemotherapy.

\section{Locoregional Recurrent NPC}

Approximately $10 \%-20 \%$ of NPC patients will develop locoregional recurrence after definitive radiotherapy. Both surgical resection and re-irradiation are potential treatment options; the former is the modality of choice if expertise is available and a clear surgical margin is achievable (69). However, the toxicity and complication rates with either approach are high, especially in elderly patients who have suboptimal premorbid conditions.

Salvage surgery for locally recurrent NPC traditionally adopts open approaches, which result in considerable functional disability and cosmetic morbidities $(70,71)$. For early local recurrences, endoscopic nasopharyngectomy is increasingly being adopted as a less invasive but equally effective salvage option $(72,73)$. In a recent multicentre randomised phase 3 trial which enrolled only patients aged $<70$, endoscopic nasopharyngectomy was shown to improve survival in selected rT1-2 or early rT3 recurrent NPC patients compared with reirradiation (3-year OS, $85.8 \%$ vs. $68.0 \%, \mathrm{p}=0.0015$ ) (74). Of note, half of the mortality in the re-irradiation arm was due to treatment complications, where it was possibly associated with the unconventional adoption of elective clinical target volumes and hypofractionated radiotherapy regimes. For operable tumours in physically fit patients, chronological age on its own should not be the sole factor to preclude salvage surgery for recurrent NPC, as case series have supported its feasibility in elderly patients beyond the age of $70(73,75)$.

For patients in whom salvage surgery is not feasible, reirradiation to the nasopharynx is a standard of care; a 5-year local control rate of $80 \%-85 \%$ has been reported for recurrent stage I-II diseases $(76,77)$. However, the cumulative radiation injury to normal tissues is associated with high rates of morbidity and mortality even in young and fit patients. In one metaanalysis, a pooled grade- 5 toxicity rate of up to $33 \%$ has been reported (78); lethal pharyngeal mucositis, nasal haemorrhage, or radiation encephalopathy was observed despite stringent eligibility criteria in a clinical trial setting (74). Old age is an adverse prognostic factor for survival and an independent risk factor for lethal nasopharyngeal necrosis after NPC reirradiation $(75,79-81)$. Extra caution on patient selection is required before starting elderly patients with radical reirradiation. Apart from pretreatment CGA as in primary radiotherapy, other factors to consider include the time interval from primary radiotherapy, tumour volume, rT classification, and the severity of preexisting late toxicities (80). There is yet no concrete evidence on the benefit of integrating systemic therapy to re-irradiation, but extrapolating from the studies on primary treatment, majority of centres would recommend combined modality for locoregionally advanced recurrent NPC (69). Patient tolerance to chemotherapy is typically poor in the re-irradiation setting, with a high rate of discontinuation and treatment-related toxicities even in young individuals (82). The exact risk-benefit ratio is even more uncertain for elderly patients; routine addition of chemotherapy is generally not recommended.

\section{Metastatic NPC}

In the current era of IMRT which offers excellent locoregional control, distant metastasis has become the major mode of relapse for localised NPC (3). In addition, approximately 10\% of NPC patients present with de novo distant metastases (83). For platinum-sensitive metastatic disease, first-line palliative chemotherapy of choice is gemcitabine and cisplatin, which has a high response rate of $64 \%$ and confers a median progression-free survival of 7 months (84). However, this regimen is associated with a $43 \%$ rate of grade $3-4$ toxicities, which were predominantly hematologic. Given the limited marrow and renal reserve in elderly patients, delivery of this cisplatin-based doublet represents a clinical challenge. Recent randomized evidence has demonstrated further improvement in PFS with the addition of camrelizumab to gemcitabine and cisplatin; however, whether elderly patients could tolerate this intensive treatment remains questionable (85). Alternatively, monotherapy such as gemcitabine, capecitabine, and docetaxel had reported response rates ranging between $24 \%$ and $48 \%$ (86-89). The choices among these active agents should be individualized by weighing the differences in side effect profiles, patient comorbidities, renal versus hepatic reserves, and the route and frequency of administration. For patients with favourable response to platinum-doublet chemotherapy, a randomized trial has demonstrated additional survival advantage 
with sequential high-dose locoregional radiotherapy (90). Whether elderly patients can derive the same benefit remains uncertain, especially if dose reduction, substitution by carboplatin, and/or de-escalation to monotherapy has to be adopted. Given their high vulnerability to acute radiation toxicities, sequential locoregional therapy should only be considered in highly selective elderly patients who have exceptional fitness and have achieved excellent tumour response to induction chemotherapy.

Recently, immune checkpoint inhibition has also emerged as another key pillar in palliative management of metastatic NPC. Multiple agents including pembrolizumab, nivolumab, camrelizumab, and toripalimab have demonstrated encouraging activities (91-95). Safety profiles of these agents were favourable, with grade 3-4 immune-related toxicities of approximately $10 \%$. Elderly patients, however, were again underrepresented in all these trials. While tolerance to immune checkpoint inhibition in elderly patients was shown to be similar to younger individuals, efficacy may be lower in elderly patients due to the deteriorating immune system (96). Current clinical evidence on immune checkpoint inhibitors in NPC is emerging; subgroup analyses in comparative phase III trials may help to clarify this issue in NPC.

\section{FUTURE DIRECTIONS - INTEGRATING GERIATRIC ASSESSMENT/ INTERVENTIONS INTO THE MANAGEMENT OF NPC IN ELDERLIES}

Despite the new advances in the management of NPC, elderly patients were often underrepresented in clinical trials. Elderly patients are of a heterogeneous group, ranging from fit to frail, and individualised decisions are always needed. There are emerging data showing that a combination of geriatric interventions can improve clinical outcomes. The randomised controlled trial by Li et al. randomised 613 patients aged 65 years or older planned for new chemotherapy into multidisciplinary geriatric assessment-driven intervention (GAIN) and standardof-care (SOC) arms (97). Patients in the GAIN arm had a lower incidence of grade 3 or higher chemotherapy-related toxic effects compared with the SOC arm (50.5\% vs. $60.6 \%, \mathrm{p}=0.02)$. There was also a significant increase in the completion of advance directives in the GAIN arm $(28.4 \%$ vs. $13.3 \%, \mathrm{p}<0.001)$. Another study by Mohile et al. which included 718 patients aged 70 years or older with advanced cancer demonstrated that integration of geriatric interventions into usual care reduced the incidence of grade $3-5$ toxicities ( $51 \%$ vs. $71 \%, \mathrm{p}=0.0001)$ and incidence of falls ( $12 \%$ vs. $21 \%, \mathrm{p}=0.0035)(98)$. These studies demonstrated that the integration of geriatric interventions in cancer management in elderly patients certainly improved the clinical outcome and reduced toxicities.

A multicentre, randomised controlled trial on the impact of CGA on survival, function, and nutritional status in elderly patients with head and neck cancer (EGeSOR; Trial registration: NCT02025062) is now ongoing in 13 sites in France (99). The interventions in the study include 1) the CGA conducted by a geriatrician before cancer treatment, 2) participation of the same geriatrician in cancer treatment selection, 3) a standardised geriatric therapeutic intervention designed by the same geriatrician, and 4) geriatric follow-up for 24 months. The results of this study to demonstrate the direct clinical benefit of CGA on outcomes of older patients with head and neck cancer are eagerly awaited.

Besides optimizing the reversible condition with geriatric interventions, individualised treatment plans for fit and unfit elderly patients need to be considered. There are studies investigating if escalating or de-escalating treatment regimen is suitable for older patients. For example, the ongoing phase 3 GORTEC ELAN-RT trial (NCT01864850) compares the efficacy and safety of standard radiotherapy (70 Gy in 35 fractions over 7 weeks) with hypofractionated split course radiotherapy (30 Gy/10 fractions, with a 2-week gap for toxicity management, followed by $25 \mathrm{~Gy} / 10$ fractions) in vulnerable elderly patients with head and neck squamous cell carcinoma planned for radical radiotherapy (100). Another prospective study, the ELAN (Elderly head and Neck cancer) FIT and UNFIT, which was a prospective study, first classified patients aged 70 or above with recurrent or metastatic head and neck squamous cell cancer as fit and unfit (101). Unfit patients were randomised to weekly methotrexate or biweekly cetuximab. The study showed that patients with performance status ECOG 2 did not benefit from systemic treatment, and there was no efficacy difference in methotrexate and cetuximab. Similar studies on individualised management plans for elderly patients with NPC, who form a heterogeneous group from fit to frail, are warranted.

\section{CONCLUSION}

In conclusion, elderly patients form a heterogeneous group in terms of their health condition, performance status, physical reserve, and social support. Unfortunately, there is a paucity of data and lack of guidelines on the management of NPC in elderly people. Moreover, most of the existing data are non-randomized. In all elderly NPC patients, pretreatment evaluation with geriatric assessment is essential to evaluate their comorbidities, physical functioning, psychological well-being, and social support. Efforts should be taken to identify risk factors for treatment-related toxicities, and modifiable factors should be controlled before treatment. Currently, similar to other adult NPC patients, IMRT is the standard of treatment in elderly NPC patients. The decision on adding chemotherapy concurrently with RT should be considered carefully because of the limited efficacy data and potential toxicities from chemotherapy. In a metastatic setting, the choice of anticancer treatment should be individualized according to the patients' preference, general condition, and comorbidities. A multidisciplinary approach, early palliative care and good communication on treatment goals are essential to support the elderly patients and their families through the treatment pathway. 


\section{AUTHOR CONTRIBUTIONS}

WC, JC, and WN contributed to the article writing. WK, AL, JL, and ZX edited the article. AL contributed the idea of this article writing. All authors contributed to the article and approved the submitted version.

\section{REFERENCES}

1. Hassett MJ, Rao SR, Brozovic S, Stahl JE, Schwartz JH, Maloney B, et al. Chemotherapy-Related Hospitalization Among Community Cancer Center Patients. Oncologist (2011) 16(3):378-87. doi: 10.1634/theoncologist.2010-0354

2. Muss HB, Woolf S, Berry D, Cirrincione C, Weiss RB, Budman D, et al. Adjuvant Chemotherapy in Older and Younger Women With Lymph Node-Positive Breast Cancer. JAMA (2005) 293(9):1073-81. doi: 10.1001/ jama.293.9.1073

3. Sargent DJ, Goldberg RM, Jacobson SD, Macdonald JS, Labianca R, Haller DG, et al. A Pooled Analysis of Adjuvant Chemotherapy for Resected Colon Cancer in Elderly Patients. N Engl J Med (2001) 345(15):1091-7. doi: 10.1056/NEJMoa010957

4. Dees EC, O’Reilly S, Goodman SN, Sartorius S, Levine MA, Jones RJ, et al. A Prospective Pharmacologic Evaluation of Age-Related Toxicity of Adjuvant Chemotherapy in Women With Breast Cancer. Cancer Invest (2000) 18 (6):521-9. doi: 10.3109/07357900009012191

5. Zhang Y, Yi JL, Huang XD, Xu GZ, Xiao JP, Li SY, et al. Inherently Poor Survival of Elderly Patients With Nasopharyngeal Carcinoma. Head Neck (2015) 37(6):771-6. doi: 10.1002/hed.23497

6. Sze HC, Ng WT, Chan OS, Shum TC, Chan LL, Lee AW. Radical Radiotherapy for Nasopharyngeal Carcinoma in Elderly Patients: The Importance of Co-Morbidity Assessment. Oral Oncol (2012) 48(2):162-7. doi: 10.1016/j.oraloncology.2011.08.019

7. Hong Kong Cancer Registry. Hong Kong Cancer Registry 2018 Report. Hong Kong: Hong Kong Cancer Registry, Hospital Authority (2020).

8. Huang Y, Chen W, Haque W, Verma V, Xing Y, Teh BS, et al. The Impact of Comorbidity on Overall Survival in Elderly Nasopharyngeal Carcinoma Patients: A National Cancer Data Base Analysis. Cancer Med (2018) 7 (4):1093-101. doi: 10.1002/cam4.1377

9. Sommat K, Yit NLF, Wang F, Lim JHC. Impact of Comorbidity on Tolerability and Survival Following Curative Intent Intensity Modulated Radiotherapy in Older Patients With Nasopharyngeal Cancer. J Geriatr Oncol (2018) 9(4):352-8. doi: 10.1016/j.jgo.2018.01.006

10. Liu H, Chen QY, Guo L, Tang LQ, Mo HY, Zhong ZL, et al. Feasibility and Efficacy of Chemoradiotherapy for Elderly Patients With Locoregionally Advanced Nasopharyngeal Carcinoma: Results From a Matched Cohort Analysis. Radiat Oncol (2013) 8:70. doi: 10.1186/1748-717X-8-70

11. Liang Y, Chen KH, Yang J, Zhang J, Peng RR, Qu S, et al. Acute Toxicities and Prognosis of Elderly Patients With Nasopharyngeal Carcinoma After Intensity-Modulated Radiotherapy: Prediction With Nomogram. Cancer Manag Res (2020) 12:8821-32. doi: 10.2147/CMAR.S261717

12. Blanchard P, Lee AWM, Carmel A, Tong NW, Ma J, Chan ATC, et al. MetaAnalysis of Chemotherapy in Nasopharynx Carcinoma (MAC-NPC): An Update on 26 Trials and 7080 Patients. Clin Transl Radiat Oncol (2022) 32:59-68. doi: 10.1016/j.ctro.2021.11.007

13. Lin JH, Wen CP, Jiang CQ, Yuan JM, Chen CJ, Ho SY, et al. Smoking and Nasopharyngeal Cancer: Individual Data Meta-Analysis of Six Prospective Studies on 334935 Men. Int J Epidemiol (2021) 50(3):975-86. doi: 10.1093/ ije/dyab060

14. Ramakrishnan Y, Paleri V, Shah R, Steen IN, Wight RG, Kelly CG. Comorbidity in Nasopharyngeal Carcinoma: A Preliminary Communication on the Prevalence, Descriptive Distribution and Impact on Outcome. Clin Otolaryngol (2007) 32(6):484-8. doi: 10.1111/j.17494486.2007.01543.x

15. Guo R, Chen XZ, Chen L, Jiang F, Tang LL, Mao YP, et al. Comorbidity Predicts Poor Prognosis in Nasopharyngeal Carcinoma: Development and Validation of a Predictive Score Model. Radiother Oncol (2015) 114(2):24956. doi: 10.1016/j.radonc.2014.12.002

\section{SUPPLEMENTARY MATERIAL}

The Supplementary Material for this article can be found online at: https://www.frontiersin.org/articles/10.3389/fonc.2022.810690/ full\#supplementary-material

16. Kallogjeri D, Gaynor SM, Piccirillo ML, Jean RA, Spitznagel EL Jr, Piccirillo JF. Comparison of Comorbidity Collection Methods. J Am Coll Surg (2014) 219(2):245-55. doi: 10.1016/j.jamcollsurg.2014.01.059

17. Jin YN, Zhang WJ, Cai XY, Li MS, Lawrence WR, Wang SY, et al. The Characteristics and Survival Outcomes in Patients Aged 70 Years and Older With Nasopharyngeal Carcinoma in the Intensity-Modulated Radiotherapy Era. Cancer Res Treat (2019) 51(1):34-42. doi: 10.4143/crt.2017.551

18. Yang CC, Chen PC, Hsu CW, Chang SL, Lee CC. Validity of the AgeAdjusted Charlson Comorbidity Index on Clinical Outcomes for Patients With Nasopharyngeal Cancer Post Radiation Treatment: A 5-Year Nationwide Cohort Study. PLoS One (2015) 10(1):e0117323. doi: 10.1371/ journal.pone.0117323

19. Wildiers H, Heeren P, Puts M, Topinkova E, Janssen-Heijnen ML, Extermann $\mathrm{M}$, et al. International Society of Geriatric Oncology Consensus on Geriatric Assessment in Older Patients With Cancer. J Clin Oncol (2014) 32(24):2595-603. doi: 10.1200/JCO.2013.54.8347

20. Mohile SG, Dale W, Somerfield MR, Hurria A. Practical Assessment and Management of Vulnerabilities in Older Patients Receiving Chemotherapy: ASCO Guideline for Geriatric Oncology Summary. J Oncol Pract (2018) 14 (7):442-6. doi: 10.1200/JOP.18.00180

21. Mohile SG, Velarde C, Hurria A, Magnuson A, Lowenstein L, Pandya C, et al. Geriatric Assessment-Guided Care Processes for Older Adults: A Delphi Consensus of Geriatric Oncology Experts. J Natl Compr Canc Netw (2015) 13(9):1120-30. doi: 10.6004/jnccn.2015.0137

22. Salas S, Deville JL, Giorgi R, Pignon T, Bagarry D, Barrau K, et al. Nutritional Factors as Predictors of Response to Radio-Chemotherapy and Survival in Unresectable Squamous Head and Neck Carcinoma. Radiother Oncol (2008) 87(2):195-200. doi: 10.1016/j.radonc.2008.02.011

23. Li R, Su L, Hua Y, Ye JR, Song XR, Tian J, et al. Anxiety and Depression Status Prior to Radiotherapy in Patients With Nasopharyngeal Carcinoma and its Effect on Acute Radiation Toxicities. Eur J Cancer Care (Engl) (2021) 30(6):e13487. doi: 10.1111/ecc.13487

24. Garcia MV, Agar MR, Soo WK, To T, Phillips JL. Screening Tools for Identifying Older Adults With Cancer Who May Benefit From a Geriatric Assessment: A Systematic Review. JAMA Oncol (2021) 7(4):616-27. doi: 10.1001/jamaoncol.2020.6736

25. Pottel L, Lycke M, Boterberg T, Pottel H, Goethals L, Duprez F, et al. G-8 Indicates Overall and Quality-Adjusted Survival in Older Head and Neck Cancer Patients Treated With Curative Radiochemotherapy. BMC Cancer (2015) 15:875. doi: 10.1186/s12885-015-1800-1

26. Ishii R, Ogawa T, Ohkoshi A, Nakanome A, Takahashi M, Katori Y. Use of the Geriatric- 8 Screening Tool to Predict Prognosis and Complications in Older Adults With Head and Neck Cancer: A Prospective, Observational Study. J Geriatr Oncol (2021) 12(7):1039-43. doi: 10.1016/j.jgo.2021.03.008

27. Au KH, Ngan RKC, Ng AWY, Poon DMC, Ng WT, Yuen KT, et al. Treatment Outcomes of Nasopharyngeal Carcinoma in Modern Era After Intensity Modulated Radiotherapy (IMRT) in Hong Kong: A Report of 3328 Patients (HKNPCSG 1301 Study). Oral Oncol (2018) 77:16-21. doi: 10.1016/ j.oraloncology.2017.12.004

28. Peng G, Wang T, Yang KY, Zhang S, Zhang T, Li Q, et al. A Prospective, Randomized Study Comparing Outcomes and Toxicities of IntensityModulated Radiotherapy vs. Conventional Two-Dimensional Radiotherapy for the Treatment of Nasopharyngeal Carcinoma. Radiother Oncol (2012) 104(3):286-93. doi: 10.1016/j.radonc.2012.08.013

29. Lee AW, Ng WT, Chan LL, Hung WM, Chan CC, Sze HC, et al. Evolution of Treatment for Nasopharyngeal Cancer-Success and Setback in the IntensityModulated Radiotherapy Era. Radiother Oncol (2014) 110(3):377-84. doi: 10.1016/j.radonc.2014.02.003 
30. Lin J-C, Han L, Lin S, Li R, Xu Y, Pan J. Therapeutic Effect of Radiotherapy and Chemotherapy in 202 Elderly Patients With Nasopharyngeal Carcinoma. Chin J Radiat Oncol (2013) 22:461-4. doi: 10.3760/ cma.j.issn.1004-4221.2013.06.011

31. Chan SH, Ng WT, Kam KL, Lee MC, Choi CW, Yau TK, et al. Sensorineural Hearing Loss After Treatment of Nasopharyngeal Carcinoma: A Longitudinal Analysis. Int J Radiat Oncol Biol Phys (2009) 73(5):1335-42. doi: 10.1016/j.ijrobp.2008.07.034

32. McDowell LJ, Rock K, Xu W, Chan B, Waldron J, Lu L, et al. Long-Term Late Toxicity, Quality of Life, and Emotional Distress in Patients With Nasopharyngeal Carcinoma Treated With Intensity Modulated Radiation Therapy. Int J Radiat Oncol Biol Phys (2018) 102(2):340-52. doi: 10.1016/ j.ijrobp.2018.05.060

33. McDowell LJ, Ringash J, Xu W, Chan B, Lu L, Waldron J, et al. A Cross Sectional Study in Cognitive and Neurobehavioral Impairment in LongTerm Nasopharyngeal Cancer Survivors Treated With Intensity-Modulated Radiotherapy. Radiother Oncol (2019) 131:179-85. doi: 10.1016/ j.radonc.2018.09.012

34. Chow JCH, Cheung KM, Au KH, Zee BCY, Lee J, Ngan RKC, et al. Radiation-Induced Hypoglossal Nerve Palsy After Definitive Radiotherapy for Nasopharyngeal Carcinoma: Clinical Predictors and Dose-Toxicity Relationship. Radiother Oncol (2019) 138:93-8. doi: 10.1016/ j.radonc.2019.06.011

35. Luk YS, Shum JS, Sze HC, Chan LL, Ng WT, Lee AW. Predictive Factors and Radiological Features of Radiation-Induced Cranial Nerve Palsy in Patients With Nasopharyngeal Carcinoma Following Radical Radiotherapy. Oral Oncol (2013) 49(1):49-54. doi: 10.1016/j.oraloncology.2012.07.011

36. Fang FM, Tsai WL, Lee TF, Liao KC, Chen HC, Hsu HC. Multivariate Analysis of Quality of Life Outcome for Nasopharyngeal Carcinoma Patients After Treatment. Radiother Oncol (2010) 97(2):263-9. doi: 10.1016/ j.radonc.2010.05.022

37. Hong JS, Tian J, Han QF, Ni QY. Quality of Life of Nasopharyngeal Cancer Survivors in China. Curr Oncol (2015) 22(3):e142-7. doi: 10.3747/co.22.2323

38. Zhai RP, Kong FF, Du CR, Hu CS, Ying HM. Radiation-Induced Hypothyroidism After IMRT for Nasopharyngeal Carcinoma: Clinical and Dosimetric Predictors in a Prospective Cohort Study. Oral Oncol (2017) 68:44-9. doi: 10.1016/j.oraloncology.2017.03.005

39. Sommat K, Ong WS, Hussain A, Soong YL, Tan T, Wee J, et al. Thyroid V40 Predicts Primary Hypothyroidism After Intensity Modulated Radiation Therapy for Nasopharyngeal Carcinoma. Int J Radiat Oncol Biol Phys (2017) 98(3):574-80. doi: 10.1016/j.ijrobp.2017.03.007

40. Lee TF, Liou MH, Ting HM, Chang L, Lee HY, Wan Leung S, et al. Patientand Therapy-Related Factors Associated With the Incidence of Xerostomia in Nasopharyngeal Carcinoma Patients Receiving Parotid-Sparing Helical Tomotherapy. Sci Rep (2015) 5:13165. doi: 10.1038/srep13165

41. Chow JCH, Tam AHP, Cheung KM, Lee VHF, Chiang CL, Tong M, et al. Second Primary Cancer After Intensity-Modulated Radiotherapy for Nasopharyngeal Carcinoma: A Territory-Wide Study by HKNPCSG. Oral Oncol (2020) 111:105012. doi: 10.1016/j.oraloncology.2020.105012

42. Lee AW, Ng WT, Pan JJ, Poh SS, Ahn YC, AlHussain H, et al. International Guideline for the Delineation of the Clinical Target Volumes (CTV) for Nasopharyngeal Carcinoma. Radiother Oncol (2018) 126(1):25-36. doi: 10.1016/j.radonc.2017.10.032

43. Lee AW, Ng WT, Pan JJ, Chiang CL, Poh SS, Choi HC, et al. International Guideline on Dose Prioritization and Acceptance Criteria in Radiation Therapy Planning for Nasopharyngeal Carcinoma. Int J Radiat Oncol Biol Phys (2019) 105(3):567-80. doi: 10.1016/j.ijrobp.2019.06.2540

44. Miao J, Wang L, Zhu M, Xiao W, Wu H, Di M, et al. Reprint of Long-Term Survival and Late Toxicities of Elderly Nasopharyngeal Carcinoma (NPC) Patients Treated by High-Total- and Fractionated-Dose Simultaneous Modulated Accelerated Radiotherapy With or Without Chemotherapy. Oral Oncol (2019) 90:126-33. doi: 10.1016/j.oraloncology.2019.01.005

45. Lewis GD, Holliday EB, Kocak-Uzel E, Hernandez M, Garden AS, Rosenthal DI, et al. Intensity-Modulated Proton Therapy for Nasopharyngeal Carcinoma: Decreased Radiation Dose to Normal Structures and Encouraging Clinical Outcomes. Head Neck (2016) 38(Suppl 1):E1886-95. doi: 10.1002/hed.24341
46. Holliday EB, Garden AS, Rosenthal DI, Fuller CD, Morrison WH, Gunn GB, et al. Proton Therapy Reduces Treatment-Related Toxicities for Patients With Nasopharyngeal Cancer: A Case-Match Control Study of IntensityModulated Proton Therapy and Intensity-Modulated Photon Therapy. Int $J$ Particle Ther (2015) 2(1):19-28. doi: 10.14338/ijpt-15-00011.1

47. Chen YP, Ismaila N, Chua MLK, Colevas AD, Haddad R, Huang SH, et al. Chemotherapy in Combination With Radiotherapy for Definitive-Intent Treatment of Stage II-IVA Nasopharyngeal Carcinoma: CSCO and ASCO Guideline. J Clin Oncol (2021) 39(7):840-59. doi: 10.1200/JCO.20.03237

48. Bossi P, Chan AT, Licitra L, Trama A, Orlandi E, Hui EP, et al. Nasopharyngeal Carcinoma: ESMO-EURACAN Clinical Practice Guidelines for Diagnosis, Treatment and Follow-Up(Dagger). Ann Oncol (2021) 32(4):452-65. doi: 10.1016/j.annonc.2020.12.007

49. Zeng Q, Xiang YQ, Wu PH, Lv X, Qian CN, Guo X. A Matched Cohort Study of Standard Chemo-Radiotherapy Versus Radiotherapy Alone in Elderly Nasopharyngeal Carcinoma Patients. PLoS One (2015) 10(3): e0119593. doi: 10.1371/journal.pone.0119593

50. Lacas B, Carmel A, Landais C, Wong SJ, Licitra L, Tobias JS, et al. MetaAnalysis of Chemotherapy in Head and Neck Cancer (MACH-NC): An Update on 107 Randomized Trials and 19,805 Patients, on Behalf of MACHNC Group. Radiother Oncol (2021) 156:281-93. doi: 10.1016/j.radonc. 2021.01.013

51. Lyu Y, Ni M, Zhai R, Kong F, Du C, Hu C, et al. Clinical Characteristics and Prognosis of Elderly Nasopharyngeal Carcinoma Patients Receiving Intensity-Modulated Radiotherapy. Eur Arch Otorhinolaryngol (2021) 278 (7):2549-57. doi: 10.1007/s00405-020-06399-5

52. Ahn MJ, D'Cruz A, Vermorken JB, Chen JP, Chitapanarux I, Dang HQ, et al. Clinical Recommendations for Defining Platinum Unsuitable Head and Neck Cancer Patient Populations on Chemoradiotherapy: A Literature Review. Oral Oncol (2016) 53:10-6. doi: 10.1016/j.oraloncology.2015.11.019

53. Chan AT, Teo PM, Ngan RK, Leung TW, Lau WH, Zee B, et al. Concurrent Chemotherapy-Radiotherapy Compared With Radiotherapy Alone in Locoregionally Advanced Nasopharyngeal Carcinoma: Progression-Free Survival Analysis of a Phase III Randomized Trial. J Clin Oncol (2002) 20 (8):2038-44. doi: 10.1200/JCO.2002.08.149

54. Chen QY, Wen YF, Guo L, Liu H, Huang PY, Mo HY, et al. Concurrent Chemoradiotherapy vs Radiotherapy Alone in Stage II Nasopharyngeal Carcinoma: Phase III Randomized Trial. J Natl Cancer Inst (2011) 103 (23):1761-70. doi: 10.1093/jnci/djr432

55. Liang H, Xia W-X, Lv X, Sun R, Zeng Q, Li S-W, et al. Concurrent Chemoradiotherapy With 3-Weekly Versus Weekly Cisplatin in Patients With Locoregionally Advanced Nasopharyngeal Carcinoma: A Phase 3 Multicentre Randomised Controlled Trial (ChiCTR-TRC-12001979). J Clin Oncol (2017) 35(15_suppl):6006. doi: 10.1200/JCO.2017.35.15_suppl.6006

56. Chitapanarux I, Lorvidhaya V, Kamnerdsupaphon P, Sumitsawan Y, Tharavichitkul E, Sukthomya V, et al. Chemoradiation Comparing Cisplatin Versus Carboplatin in Locally Advanced Nasopharyngeal Cancer: Randomised, Non-Inferiority, Open Trial. Eur J Cancer (2007) 43 (9):1399-406. doi: 10.1016/j.ejca.2007.03.022

57. Tang LQ, Chen DP, Guo L, Mo HY, Huang Y, Guo SS, et al. Concurrent Chemoradiotherapy With Nedaplatin Versus Cisplatin in Stage II-IVB Nasopharyngeal Carcinoma: An Open-Label, Non-Inferiority, Randomised Phase 3 Trial. Lancet Oncol (2018) 19(4):461-73. doi: 10.1016/S1470-2045 (18)30104-9

58. Lv X, Cao X, Xia WX, Liu KY, Qiang MY, Guo L, et al. Induction Chemotherapy With Lobaplatin and Fluorouracil Versus Cisplatin and Fluorouracil Followed by Chemoradiotherapy in Patients With Stage IIIIVB Nasopharyngeal Carcinoma: An Open-Label, Non-Inferiority, Randomised, Controlled, Phase 3 Trial. Lancet Oncol (2021) 22(5):716-26. doi: 10.1016/S1470-2045(21)00075-9

59. Wang F, Jiang C, Ye Z, Sun Q, Liu T, Xu M, et al. Efficacy and Safety of Nimotuzumab Plus Radiotherapy With or Without Cisplatin-Based Chemotherapy in an Elderly Patient Subgroup (Aged 60 and Older) With Nasopharyngeal Carcinoma. Transl Oncol (2018) 11(2):338-45. doi: 10.1016/j.tranon.2018.01.013

60. Al-Sarraf M, LeBlanc M, Giri PG, Fu KK, Cooper J, Vuong T, et al. Chemoradiotherapy Versus Radiotherapy in Patients With Advanced 
Nasopharyngeal Cancer: Phase III Randomized Intergroup Study 0099. J Clin Oncol (1998) 16(4):1310-7. doi: 10.1200/JCO.1998.16.4.1310

61. Wee J, Tan EH, Tai BC, Wong HB, Leong SS, Tan T, et al. Randomized Trial of Radiotherapy Versus Concurrent Chemoradiotherapy Followed by Adjuvant Chemotherapy in Patients With American Joint Committee on Cancer/International Union Against Cancer Stage III and IV Nasopharyngeal Cancer of the Endemic Variety. J Clin Oncol (2005) 23 (27):6730-8. doi: 10.1200/JCO.2005.16.790

62. Lee AW, Lau WH, Tung SY, Chua DT, Chappell R, Xu L, et al. Preliminary Results of a Randomized Study on Therapeutic Gain by Concurrent Chemotherapy for Regionally-Advanced Nasopharyngeal Carcinoma: NPC-9901 Trial by the Hong Kong Nasopharyngeal Cancer Study Group. J Clin Oncol (2005) 23(28):6966-75. doi: 10.1200/JCO.2004. 00.7542

63. Lee AW, Tung SY, Chan AT, Chappell R, Fu YT, Lu TX, et al. A Randomized Trial on Addition of Concurrent-Adjuvant Chemotherapy and/or Accelerated Fractionation for Locally-Advanced Nasopharyngeal Carcinoma. Radiother Oncol (2011) 98(1):15-22. doi: 10.1016/ j.radonc.2010.09.023

64. Chen YP, Liu X, Zhou Q, Yang KY, Jin F, Zhu XD, et al. Metronomic Capecitabine as Adjuvant Therapy in Locoregionally Advanced Nasopharyngeal Carcinoma: A Multicentre, Open-Label, Parallel-Group, Randomised, Controlled, Phase 3 Trial. Lancet (2021) 398(10297):303-13. doi: 10.1016/S0140-6736(21)01123-5

65. Zhang Y, Chen L, Hu GQ, Zhang N, Zhu XD, Yang KY, et al. Gemcitabine and Cisplatin Induction Chemotherapy in Nasopharyngeal Carcinoma. $N$ Engl J Med (2019) 381(12):1124-35. doi: 10.1056/NEJMoa1905287

66. Sun Y, Li WF, Chen NY, Zhang N, Hu GQ, Xie FY, et al. Induction Chemotherapy Plus Concurrent Chemoradiotherapy Versus Concurrent Chemoradiotherapy Alone in Locoregionally Advanced Nasopharyngeal Carcinoma: A Phase 3, Multicentre, Randomised Controlled Trial. Lancet Oncol (2016) 17(11):1509-20. doi: 10.1016/S1470-2045(16)30410-7

67. Yang Q, Cao SM, Guo L, Hua YJ, Huang PY, Zhang XL, et al. Induction Chemotherapy Followed by Concurrent Chemoradiotherapy Versus Concurrent Chemoradiotherapy Alone in Locoregionally Advanced Nasopharyngeal Carcinoma: Long-Term Results of a Phase III Multicentre Randomised Controlled Trial. Eur J Cancer (2019) 119:87-96. doi: 10.1016/ j.ejca.2019.07.007

68. Wang C, Tang X, Wang J, Song J, Xu Y. Induction Chemotherapy Plus Concurrent Chemoradiotherapy vs Concurrent Chemoradiotherapy in Elderly Patients With Advanced Nasopharyngeal Carcinoma. Otolaryngol Head Neck Surg (2017) 157(2):233-8. doi: 10.1177/0194599 817699402

69. Ng WT, Soong YL, Ahn YC, AlHussain H, Choi HCW, Corry J, et al. International Recommendations on Reirradiation by Intensity Modulated Radiation Therapy for Locally Recurrent Nasopharyngeal Carcinoma. Int $J$ Radiat Oncol Biol Phys (2021) 110(3):682-95. doi: 10.1016/ j.ijrobp.2021.01.041

70. King WW, Ku PK, Mok CO, Teo PM. Nasopharyngectomy in the Treatment of Recurrent Nasopharyngeal Carcinoma: A Twelve-Year Experience. Head Neck (2000) 22(3):215-22. doi: 10.1002/(sici)1097-0347(200005)22:3<215:: aid-hed2>3.0.co;2-b

71. Fee WE Jr, Moir MS, Choi EC, Goffinet D. Nasopharyngectomy for Recurrent Nasopharyngeal Cancer: A 2- to 17-Year Follow-Up. Arch Otolaryngol Head Neck Surg (2002) 128(3):280-4. doi: 10.1001/ archotol.128.3.280

72. Emanuelli E, Albu S, Cazzador D, Pedruzzi B, Babighian G, Martini A. Endoscopic Surgery for Recurrent Undifferentiated Nasopharyngeal Carcinoma. J Craniofac Surg (2014) 25(3):1003-8. doi: 10.1097/ SCS.0000000000000698

73. Chen MY, Wen WP, Guo X, Yang AK, Qian CN, Hua YJ, et al. Endoscopic Nasopharyngectomy for Locally Recurrent Nasopharyngeal Carcinoma. Laryngoscope (2009) 119(3):516-22. doi: 10.1002/lary.20133

74. Liu YP, Wen YH, Tang J, Wei Y, You R, Zhu XL, et al. Endoscopic Surgery Compared With Intensity-Modulated Radiotherapy in Resectable Locally Recurrent Nasopharyngeal Carcinoma: A Multicentre, Open-Label, Randomised, Controlled, Phase 3 Trial. Lancet Oncol (2021) 22(3):381-90. doi: $10.1016 /$ S1470-2045(20)30673-2
75. Chan JY, To VS, Chow VL, Wong ST, Wei WI. Multivariate Analysis of Prognostic Factors for Salvage Nasopharyngectomy via the Maxillary Swing Approach. Head Neck (2014) 36(7):1013-7. doi: 10.1002/hed.23403

76. Han F, Zhao C, Huang SM, Lu LX, Huang Y, Deng XW, et al. Long-Term Outcomes and Prognostic Factors of Re-Irradiation for Locally Recurrent Nasopharyngeal Carcinoma Using Intensity-Modulated Radiotherapy. Clin Oncol (R Coll Radiol) (2012) 24(8):569-76. doi: 10.1016/j.clon.2011.11.010

77. Hua YJ, Han F, Lu LX, Mai HQ, Guo X, Hong MH, et al. Long-Term Treatment Outcome of Recurrent Nasopharyngeal Carcinoma Treated With Salvage Intensity Modulated Radiotherapy. Eur J Cancer (2012) 48 (18):3422-8. doi: 10.1016/j.ejca.2012.06.016

78. Leong YH, Soon YY, Lee KM, Wong LC, Tham IWK, Ho FCH. Long-Term Outcomes After Reirradiation in Nasopharyngeal Carcinoma With Intensity-Modulated Radiotherapy: A Meta-Analysis. Head Neck (2018) 40 (3):622-31. doi: 10.1002/hed.24993

79. Chan OS, Sze HC, Lee MC, Chan LL, Chang AT, Lee SW, et al. Reirradiation With Intensity-Modulated Radiotherapy for Locally Recurrent T3 to T4 Nasopharyngeal Carcinoma. Head Neck (2017) 39(3):533-40. doi: 10.1002/ hed. 24645

80. Li YQ, Tian YM, Tan SH, Liu MZ, Kusumawidjaja G, Ong EHW, et al. Prognostic Model for Stratification of Radioresistant Nasopharynx Carcinoma to Curative Salvage Radiotherapy. J Clin Oncol (2018) 36 (9):891-9. doi: 10.1200/JCO.2017.75.5165

81. Ng WT, Wong ECY, Cheung AKW, Chow JCH, Poon DMC, Lai JWY, et al. Patterns of Care and Treatment Outcomes for Local Recurrence of NPC After Definite IMRT-A Study by. Head Neck (2019) 41(10):3661-9. doi: $10.1002 /$ hed.25892

82. Ng WT, Ngan RKC, Kwong DLW, Tung SY, Yuen KT, Kam MKM, et al. Prospective, Multicenter, Phase 2 Trial of Induction Chemotherapy Followed by Bio-Chemoradiotherapy for Locally Advanced Recurrent Nasopharyngeal Carcinoma. Int J Radiat Oncol Biol Phys (2018) 100 (3):630-8. doi: 10.1016/j.ijrobp.2017.11.038

83. Chua MLK, Wee JTS, Hui EP, Chan ATC. Nasopharyngeal Carcinoma. Lancet (2016) 387(10022):1012-24. doi: 10.1016/S0140-6736(15)00055-0

84. Zhang L, Huang Y, Hong S, Yang Y, Yu G, Jia J, et al. Gemcitabine Plus Cisplatin Versus Fluorouracil Plus Cisplatin in Recurrent or Metastatic Nasopharyngeal Carcinoma: A Multicentre, Randomised, Open-Label, Phase 3 Trial. Lancet (2016) 388(10054):1883-92. doi: 10.1016/S0140-6736(16)31388-5

85. Yang Y, Qu S, Li J, Hu C, Xu M, Li W, et al. Camrelizumab Versus Placebo in Combination With Gemcitabine and Cisplatin as First-Line Treatment for Recurrent or Metastatic Nasopharyngeal Carcinoma (CAPTAIN-1st): A Multicentre, Randomised, Double-Blind, Phase 3 Trial. Lancet Oncol (2021) 22(8):1162-74. doi: 10.1016/S1470-2045(21)00302-8

86. Chua DT, Sham JS, Au GK. A Phase II Study of Capecitabine in Patients With Recurrent and Metastatic Nasopharyngeal Carcinoma Pretreated With Platinum-Based Chemotherapy. Oral Oncol (2003) 39(4):361-6. doi: $10.1016 / \mathrm{s} 1368-8375(02) 00120-3$

87. Foo KF, Tan EH, Leong SS, Wee JT, Tan T, Fong KW, et al. Gemcitabine in Metastatic Nasopharyngeal Carcinoma of the Undifferentiated Type. Ann Oncol (2002) 13(1):150-6. doi: 10.1093/annonc/mdf002

88. Zhang L, Zhang Y, Huang PY, Xu F, Peng PJ, Guan ZZ. Phase II Clinical Study of Gemcitabine in the Treatment of Patients With Advanced Nasopharyngeal Carcinoma After the Failure of Platinum-Based Chemotherapy. Cancer Chemother Pharmacol (2008) 61(1):33-8. doi: 10.1007/s00280-007-0441-8

89. Ngeow J, Lim WT, Leong SS, Ang MK, Toh CK, Gao F, et al. Docetaxel is Effective in Heavily Pretreated Patients With Disseminated Nasopharyngeal Carcinoma. Ann Oncol (2011) 22(3):718-22. doi: 10.1093/annonc/mdq425

90. You R, Liu YP, Huang PY, Zou X, Sun R, He YX, et al. Efficacy and Safety of Locoregional Radiotherapy With Chemotherapy vs Chemotherapy Alone in De Novo Metastatic Nasopharyngeal Carcinoma: A Multicenter Phase 3 Randomized Clinical Trial. JAMA Oncol (2020) 6(9):1345-52. doi: 10.1001/ jamaoncol.2020.1808

91. Hsu C, Lee SH, Ejadi S, Even C, Cohen RB, Le Tourneau C, et al. Safety and Antitumor Activity of Pembrolizumab in Patients With Programmed Death-Ligand 1-Positive Nasopharyngeal Carcinoma: Results of the KEYNOTE-028 Study. J Clin Oncol (2017) 35(36):4050-6. doi: 10.1200/ JCO.2017.73.3675 
92. Ma BBY, Lim WT, Goh BC, Hui EP, Lo KW, Pettinger A, et al. Antitumor Activity of Nivolumab in Recurrent and Metastatic Nasopharyngeal Carcinoma: An International, Multicenter Study of the Mayo Clinic Phase 2 Consortium (NCI-9742). J Clin Oncol (2018) 36(14):1412-8. doi: 10.1200/ JCO.2017.77.0388

93. Fang W, Yang Y, Ma Y, Hong S, Lin L, He X, et al. Camrelizumab (SHR-1210) Alone or in Combination With Gemcitabine Plus Cisplatin for Nasopharyngeal Carcinoma: Results From Two Single-Arm, Phase 1 Trials. Lancet Oncol (2018) 19(10):1338-50. doi: 10.1016/S1470-2045(18)30495-9

94. Wang FH, Wei XL, Feng J, Li Q, Xu N, Hu XC, et al. Efficacy, Safety, and Correlative Biomarkers of Toripalimab in Previously Treated Recurrent or Metastatic Nasopharyngeal Carcinoma: A Phase II Clinical Trial (POLARIS02). J Clin Oncol (2021) 39(7):704-12. doi: 10.1200/JCO.20.02712

95. Mai HQ, Chen QY, Chen D, Hu C, Yang K, Wen J, et al. Toripalimab or Placebo Plus Chemotherapy as First-Line Treatment in Advanced Nasopharyngeal Carcinoma: A Multicenter Randomized Phase 3 Trial. Nat Med (2021) 27(9):1536-43. doi: 10.1038/s41591-021-01444-0

96. Daste A, Domblides C, Gross-Goupil M, Chakiba C, Quivy A, Cochin V, et al. Immune Checkpoint Inhibitors and Elderly People: A Review. Eur J Cancer (2017) 82:155-66. doi: 10.1016/j.ejca.2017.05.044

97. Li D, Sun CL, Kim H, Soto-Perez-de-Celis E, Chung V, Koczywas M, et al. Geriatric Assessment-Driven Intervention (GAIN) on ChemotherapyRelated Toxic Effects in Older Adults With Cancer: A Randomized Clinical Trial. JAMA Oncol (2021) 7(11):e214158. doi: 10.1001/jamaoncol.2021.4158

98. Mohile SG, Mohamed MR, Xu H, Culakova E, Loh KP, Magnuson A, et al. Evaluation of Geriatric Assessment and Management on the Toxic Effects of Cancer Treatment (GAP70+): A Cluster-Randomised Study. Lancet (2021) 398(10314):1894-904. doi: 10.1016/S0140-6736(21)01789-X

99. Brugel L, Laurent M, Caillet P, Radenne A, Durand-Zaleski I, Martin M, et al. Impact of Comprehensive Geriatric Assessment on Survival, Function, and Nutritional Status in Elderly Patients With Head and Neck Cancer: Protocol for a Multicentre Randomised Controlled Trial (EGeSOR). BMC Cancer (2014) 14:427. doi: 10.1186/1471-2407-14-427

100. Ortholan C, Le Caer H, Mertens C, Leysalle A, Even C, Renard-Oldrini S, et al. PO-071: ELAN Program: Personalized Treatment According to Geriatric Assessment in Elderly Patients With Head \& Neck Cancer. Radiother Oncol (2015) 114:38. doi: 10.1016/S0167-8140(15)34831-3

101. Guigay J, Le Caer H, Mertens C, Ortholan C, Blot E, Peyrade YT, et al. Personalized Treatment According to Geriatric Assessment in 1st Line Recurrent and/or Metastatic (R/M) Head and Neck Squamous Cell Cancer (HNSCC) Patients Aged 70 or Over. ELAN (ELderly heAd and Neck Cancer) FIT and UNFIT Trials. Ann Oncol (2019) 30(Supplement_5). doi: 10.1093/ annonc/mdz252.002

Conflict of Interest: The authors declare that the research was conducted in the absence of any commercial or financial relationships that could be construed as a potential conflict of interest.

Publisher's Note: All claims expressed in this article are solely those of the authors and do not necessarily represent those of their affiliated organizations, or those of the publisher, the editors and the reviewers. Any product that may be evaluated in this article, or claim that may be made by its manufacturer, is not guaranteed or endorsed by the publisher.

Copyright (C) 2022 Chan, Chow, Xu, Li, Kwong, Ng and Lee. This is an open-access article distributed under the terms of the Creative Commons Attribution License (CC BY). The use, distribution or reproduction in other forums is permitted, provided the original author(s) and the copyright owner(s) are credited and that the original publication in this journal is cited, in accordance with accepted academic practice. No use, distribution or reproduction is permitted which does not comply with these terms. 